\title{
Fractal Dimension of a Random Invariant Set and Applications
}

\author{
Gang Wang and Yanbin Tang \\ School of Mathematics and Statistics, Huazhong University of Science and Technology, Wuhan, Hubei 430074, China \\ Correspondence should be addressed to Yanbin Tang; tangybhust@sina.com
}

Received 29 April 2013; Accepted 26 September 2013

Academic Editor: Erik Van Vleck

Copyright (c) 2013 G. Wang and Y. Tang. This is an open access article distributed under the Creative Commons Attribution License, which permits unrestricted use, distribution, and reproduction in any medium, provided the original work is properly cited.

We prove an abstract result on random invariant sets of finite fractal dimension. Then this result is applied to a stochastic semilinear degenerate parabolic equation and an upper bound is obtained for the random attractors of fractal dimension.

\section{Introduction}

The notion of random attractors is a generalization of the classical concept of global attractors for deterministic dynamical systems (see, e.g., [1-3]). Random attractors are compact invariant random sets attracting all the orbits. The asymptotic behavior of a random dynamical system (RDS) is captured by random attractors, which were first introduced in [4]. The existence of random attractors associated with stochastic partial differential equations has been extensively studied by many authors [4-9].

As in the deterministic case, finite dimensionality is an important property of random attractors which can be established for several random dynamical systems. In [10], Crauel and Flandoli developed a method to obtain finite Hausdorff dimension of a random invariant set. However, their assumptions are very restrictive. They proposed certain bounds on the derivative of the RDS as well as on the rate of approximation of the RDS by its derivative to hold uniformly in $\omega \in \Omega$ ( $\Omega$ denotes certain probability space). This drawback was overcome by using a "random squeezing property" in [11]. In [12], Debussche used the method involving the Lyapunov exponents (see [3]) to obtain an upper bound on the Hausdorff dimension for a random invariant set, and this method was developed in a recent paper [13] for bounding the fractal dimension of random invariant sets. Motivated by [14], we give a new criterion for the upper bound on fractal dimension of random invariant sets. This result does not require $C^{1}$ smoothness of the RDS. Therefore, it can be applied to more stochastic models. However, as mentioned in [14], the estimate based on our theorems usually turns out to be conservative.
In the next section, we formulate and prove our main abstract results. In Section 3, we apply our abstract results to the random attractor for the RDS generated by a stochastic semilinear degenerate parabolic equation and obtain an upper bound of fractal dimension of the random attractor. Throughout this paper, we denote by $\|\cdot\|_{X}$ the norm of Banach space $X$. The inner product and norm of $L^{2}(\Omega)$ are written as $(\cdot, \cdot)$ and $\|\cdot\|$, respectively. The letter $c$ denotes any positive constant which may be different from line to line even in the same line.

\section{Preliminaries and Main Results}

In this section, we give the main abstract results for the finite fractal dimension of a random invariant set. For that matter, we need some basic concepts.

Definition 1. Let $M$ be a compact set in a metric space $X$. The fractal (box-counting) dimension $\operatorname{dim}_{f} M$ of $M$ is defined by

$$
\operatorname{dim}_{f} M=\limsup _{\varepsilon \rightarrow 0} \frac{\ln n(M, \varepsilon)}{\ln (1 / \varepsilon)},
$$

where $n(M, \varepsilon)$ is the minimal number of closed balls of the radius $\varepsilon$ which cover the set $M$.

For other alternative formulations of the definition of the box-counting dimension, see Definition 3.1 in Falconer's book [15].

Definition 2. Let $X$ be a complete metric space endowed with the metric $d$ and let $M$ be a bounded closed set in $X$. Assume that $\varrho$ is a pseudometric defined on $M$. Let $B \subset M$ and $\varepsilon>0$. 
(i) A subset $\mathcal{U}$ in $B$ is said to be $(\varepsilon, \varrho)$-distinguishable if $\varrho\left(x, x^{\prime}\right)>\varepsilon$ for any $x, x^{\prime} \in \mathcal{U}, x \neq x^{\prime}$. We denote by $m_{\varrho}(B, \varepsilon)$ the maximal cardinality of an $(\varepsilon, \varrho)$ distinguishable subset of $B$.

(ii) The pseudometric $\varrho$ is said to be compact on $M$ if and only if $m_{\varrho}(M, \varepsilon)$ is finite for every $\varepsilon>0$.

(iii) For any $r>0$ we define a local $(r, \varepsilon, \varrho)$-capacity of the set $M$ by the formula

$\mathscr{C}_{\varrho}(M ; r, \varepsilon)=\sup \left\{\ln m_{\varrho}(B, \varepsilon): B \subset M, \operatorname{diam} B \leq 2 r\right\}$.

We next recall some notions related to RDS. The reader is referred to $[4-8,16]$ for more details. Let $(\Omega, \mathscr{F}, \mathbb{P})$ be a probability space and let $X$ be a Banach space.

Definition 3. (1) $\left(\Omega, \mathscr{F}, \mathbb{P},\left(\theta_{t}\right)_{t \in \mathbb{R}}\right)$ is called a metric dynamical system (MDS) if $\theta: \mathbb{R} \times \Omega \rightarrow \Omega$ is $(\mathscr{B}(\mathbb{R}) \times \mathscr{F}, \mathscr{F})$ measurable, $\theta_{0}$ is the identity on $\Omega, \theta_{s+t}=\theta_{s} \circ \theta_{t}$ for all $s$, $t \in \mathbb{R}$, and $\theta_{t} \mathbb{P}=\mathbb{P}$ for all $t \in \mathbb{R}$.

(2) An RDS on $X$ over an $\operatorname{MDS}\left(\Omega, \mathscr{F}, \mathbb{P},\left(\theta_{t}\right)_{t \in \mathbb{R}}\right)$ is a mapping $\phi: \mathbb{R}^{+} \times \Omega \times X \rightarrow X,(t, \omega, x) \mapsto \phi(t, \omega, x)$ which is $\left(\mathscr{B}\left(\mathbb{R}^{+}\right) \times \mathscr{F} \times \mathscr{B}(X), \mathscr{B}(X)\right)$-measurable and satisfies, for $\mathbb{P}$-a.s. $\omega \in \Omega$,

(a) $\phi(0, \omega, \cdot)=$ id on $X$,

(b) $\phi(t+s, \omega, \cdot)=\phi\left(t, \theta_{s} \omega, \cdot\right) \circ \phi(s, \omega, \cdot)$ (cocycle property) on $X$ for all $s, t \in \mathbb{R}^{+}$.

An RDS is said to be continuous on $X$ if $\phi(t, \omega): X \rightarrow X$ is continuous for all $t \in \mathbb{R}^{+}$and $\mathbb{P}$-a.s. $\omega \in \Omega$.

Let $\theta$ be a measure-preserving ergodic transformation on $(\Omega, \mathscr{F}, \mathbb{P})$ and let $S(\omega)$ be a family of maps from $X$ to $X$. We assume that $\mathscr{A}(\omega), \omega \in \Omega$, is a compact measurable set satisfying, for $\mathbb{P}$-a.s. $\omega \in \Omega$,

$$
S(\omega) \mathscr{A}(\omega)=\mathscr{A}(\theta \omega)
$$

Our aim is to study the fractal dimension of the sets $\mathscr{A}(\omega)$, $\omega \in \Omega$. We define a discrete $\operatorname{RDS}\left\{S^{n}, n \in \mathbb{Z}^{+}\right\}$by $S^{n}(\omega):=$ $S\left(\theta_{n-1} \omega\right) S\left(\theta_{n-2} \omega\right) \cdots S(\theta \omega) S(\omega)$. In the proof of the following theorem we keep track of the " $\varepsilon$-approximate $\nu$ volume":

$$
V_{\nu}(X, \varepsilon):=\varepsilon^{v} n(X, \varepsilon) .
$$

Our main results read as follows.

Theorem 4. Let $X$ be a Banach space, and $S(\omega)$ satisfies, for $\mathbb{P}$ a.s. $\omega \in \Omega$, the following:

(i) $S(\omega)$ is Lipschitz on $\mathscr{A}(\omega)$; that is, there exists $L>0$ independent of $\omega$ such that

$\left\|S(\omega) v_{1}-S(\omega) v_{2}\right\|_{X} \leq L\left\|v_{1}-v_{2}\right\|_{X}, \quad v_{1}, v_{2} \in \mathscr{A}(\omega)$, (ii) there exist compact seminorms $n_{1}(x), n_{2}(x)$ (independent of $\omega$ ) on $X$ such that

$$
\begin{aligned}
&\left\|S(\omega) v_{1}-S(\omega) v_{2}\right\|_{X} \leq \eta\left\|v_{1}-v_{2}\right\|_{X} \\
&+K\left[n_{1}\left(v_{1}-v_{2}\right)+n_{2}\right. \\
&\left.\quad \times\left(S(\omega) v_{1}-S(\omega) v_{2}\right)\right],
\end{aligned}
$$

for any $v_{1}, v_{2} \in \mathscr{A}(\omega)$, where $0 \leq \eta<1 / 2 e$ and $K>0$ are constants independent of $\omega$ (a seminorm $n(x)$ on $X$ is said to be compact if and only if for any bounded set $B \subset X$ there exists a sequence $\left\{x_{n}\right\} \subset B$ such that $n\left(x_{m}-x_{n}\right) \rightarrow 0$ as $\left.m, n \rightarrow \infty\right)$. Then $\mathscr{A}(\omega)$ has finite fractal dimension in $X$; that is, for $\mathbb{P}$-a.s. $\omega \in \Omega$,

$$
d_{f} \mathscr{A}(\omega) \leq \ln m_{0}\left(\frac{8 e K\left(1+L^{2}\right)^{1 / 2}}{1-2 e \eta}\right)
$$

where $m_{0}(R)$ is the maximal number of pairs $\left(x_{i}, y_{i}\right)$ in $X \times X$ possessing the properties

$$
\begin{gathered}
\left\|x_{i}\right\|_{X}^{2}+\left\|y_{i}\right\|_{X}^{2} \leq R^{2}, \\
n_{1}\left(x_{i}-x_{j}\right)+n_{2}\left(y_{i}-y_{j}\right)>1, \quad i \neq j .
\end{gathered}
$$

Proof. We set $\varrho_{\omega}(x, y)=K\left(n_{1}(x-y)+n_{2}(S(\omega) x-S(\omega) y)\right)$; then, for every $\omega \in \Omega, \varrho_{\omega}$ is compact on $\mathscr{A}(\omega)$ in the sense of Definition 2. From [14], we see that the local $\left(r, \varepsilon, \varrho_{\omega}\right)$ capacity of the set $\mathscr{A}(\omega)$ admits the estimate

$$
\begin{gathered}
\mathscr{C}_{\varrho_{\omega}}(\mathscr{A}(\omega) ; r, \varepsilon) \leq \ln m_{0}\left(\frac{2 K\left(1+L^{2}\right)^{1 / 2} r}{\varepsilon}\right), \\
\mathbb{P} \text {-a.s. } \omega,
\end{gathered}
$$

where $m_{0}(R)$ is the maximal number of pairs $\left(x_{i}, y_{i}\right)$ in $X \times X$ possessing the properties

$$
\begin{gathered}
\left\|x_{i}\right\|_{X}^{2}+\left\|y_{i}\right\|_{X}^{2} \leq R^{2}, \\
n_{1}\left(x_{i}-x_{j}\right)+n_{2}\left(y_{i}-y_{j}\right)>1, \quad i \neq j .
\end{gathered}
$$

For any fixed $\varepsilon_{0}>0$, we assume that $\left\{B_{i}: i=1, \ldots\right.$, $\left.n\left(\mathscr{A}(\omega), \varepsilon_{0}\right)\right\}$ is the minimal covering of $\mathscr{A}(\omega)$ by closed balls of the radius $\varepsilon_{0}$. Set $F_{i}=B_{i} \cap \mathscr{A}(\omega), i=1, \ldots, n\left(\mathscr{A}(\omega), \varepsilon_{0}\right)$. Let $\delta=(1 / 4 e)-(\eta / 2)$ and let $\left\{x_{j}^{i} ; j=1, \ldots, n_{i}\right\} \subset F_{i}$ be a maximal $\left(\delta \varepsilon_{0}, \varrho_{\omega}\right)$-distinguishable subset of $F_{i}$. Since $\varrho_{\omega}$ is compact, this finite set exists, and then we have

$$
\begin{gathered}
n_{i}=m_{\varrho_{\omega}}\left(F_{i}, \delta \varepsilon_{0}\right) \leq \exp \left\{C_{\varrho_{\omega}}\left(\mathscr{A}(\omega) ; \varepsilon_{0}, \delta \varepsilon_{0}\right)\right\} \\
\leq m_{0}\left(\frac{2 K\left(1+L^{2}\right)^{1 / 2}}{\delta}\right)=: e^{v}, \\
\mathscr{A}(\omega) \subset \bigcup_{i=1}^{n\left(\mathscr{A}(\omega), \varepsilon_{0}\right)} \bigcup_{j=1}^{n_{i}} B_{i, j}, \\
B_{i, j}=\left\{v \in F_{i}: \varrho_{\omega}\left(v, x_{j}^{i}\right) \leq \delta \varepsilon_{0}\right\} .
\end{gathered}
$$


Therefore,

$$
S(\omega) \mathscr{A}(\omega) \subset \bigcup_{i=1}^{n\left(\mathscr{A}(\omega), \varepsilon_{0}\right)} \bigcup_{j=1}^{n_{i}} S(\omega) B_{i, j} .
$$

For any $y_{1}, y_{2} \in B_{i, j}(\subset \mathscr{A}(\omega))$, we get from (6) that

$$
\begin{aligned}
d\left(S(\omega) y_{1}, S(\omega) y_{2}\right) & \leq \eta d\left(y_{1}, y_{2}\right)+\varrho_{\omega}\left(y_{1}, x_{j}^{i}\right)+\varrho_{\omega}\left(y_{2}, x_{j}^{i}\right) \\
& \leq 2(\eta+\delta) \varepsilon_{0} .
\end{aligned}
$$

Thus diam $S(\omega) B_{i, j} \leq 2(\eta+\delta) \varepsilon_{0}$ for any $i, j$. Therefore,

$$
\begin{aligned}
n\left(S(\omega) \mathscr{A}(\omega), 2(\eta+\delta) \varepsilon_{0}\right) & \leq n_{i} n\left(\mathscr{A}(\omega), \varepsilon_{0}\right) \\
& \leq e^{\nu} n\left(\mathscr{A}(\omega), \varepsilon_{0}\right) .
\end{aligned}
$$

For general $n \in \mathbb{N}$, replacing $\varrho_{\omega}, S(\omega)$, and $\mathscr{A}(\omega)$ by $\varrho_{\theta_{n} \omega}$, $S\left(\theta_{n} \omega\right)$, and $S^{n}(\omega) \mathscr{A}(\omega)$, respectively, in the above procedure and noting that $S^{n}(\omega) \mathscr{A}(\omega)=\mathscr{A}\left(\theta_{n} \omega\right)$, we can get that

$$
S\left(\theta_{n} \omega\right) S^{n}(\omega) \mathscr{A}(\omega) \subset \bigcup_{i=1}^{n\left(\mathscr{A}\left(\theta_{n} \omega\right), \varepsilon_{0}\right)} \bigcup_{j=1}^{n_{i}^{\prime}} S\left(\theta_{n} \omega\right) B_{i, j}^{\prime},
$$

where $n_{i}^{\prime} \leq e^{\nu}$, and

$$
\begin{aligned}
n\left(S\left(\theta_{n} \omega\right) S^{n}(\omega) \mathscr{A}(\omega), 2(\eta+\delta) \varepsilon_{0}\right) & \leq n_{i}^{\prime} n\left(\mathscr{A}\left(\theta_{n} \omega\right), \varepsilon_{0}\right) \\
& \leq e^{\nu} n\left(\mathscr{A}\left(\theta_{n} \omega\right), \varepsilon_{0}\right) .
\end{aligned}
$$

Thus,

$$
n\left(S^{n+1}(\omega) \mathscr{A}(\omega), 2(\eta+\delta) \varepsilon_{0}\right) \leq e^{\nu} n\left(S^{n}(\omega) \mathscr{A}(\omega), \varepsilon_{0}\right) .
$$

Setting $q=2(\eta+\delta)$, then by a standard induction procedure we deduce that

$$
n\left(S^{n}(\omega) \mathscr{A}(\omega), q^{n} \varepsilon_{0}\right) \leq e^{n v} n\left(\mathscr{A}(\omega), \varepsilon_{0}\right) .
$$

Multiplying (18) by $q^{n v} \varepsilon_{0}^{v}$ we get

$$
\begin{aligned}
V_{\nu}\left(S^{n}(\omega) \mathscr{A}(\omega), q^{n} \varepsilon_{0}\right) & \leq q^{n v} e^{n v} \varepsilon_{0}^{\nu} n\left(\mathscr{A}(\omega), \varepsilon_{0}\right) \\
& =\kappa^{n v} V_{\nu}\left(\mathscr{A}(\omega), \varepsilon_{0}\right),
\end{aligned}
$$

where $\kappa=q e=2(\eta+\delta) e<1$. Then we can get from the above inequality that

$$
V_{\nu}\left(\mathscr{A}\left(\theta_{n} \omega\right), q^{n} \varepsilon_{0}\right) \leq \kappa^{n \nu} V_{\nu}\left(\mathscr{A}(\omega), \varepsilon_{0}\right) .
$$

Therefore,

$$
V_{\nu}\left(\mathscr{A}(\omega), q^{n} \varepsilon_{0}\right) \leq \kappa^{n \nu} V_{\nu}\left(\mathscr{A}\left(\theta_{-n} \omega\right), \varepsilon_{0}\right) .
$$

On one hand, for the above $q$, setting $\beta=\ln q^{-1}$, then we have

$$
-(\beta+\delta) \leq \ln q \leq-(\beta-\delta), \quad \text { for any } \delta>0 .
$$

That is,

$$
\begin{array}{r}
-(\beta+\delta) k \leq \ln q^{k} \leq-(\beta-\delta) k, \\
\text { for any } k \in \mathbb{N} \text {, any } \delta>0 .
\end{array}
$$

This implies that

$$
\begin{array}{r}
\varepsilon_{0} e^{-(\beta+\delta) k} \leq q^{k} \varepsilon_{0} \leq e^{-(\beta-\delta) k} \varepsilon_{0}, \\
\text { for any } k \in \mathbb{N} \text {, any } \delta>0 .
\end{array}
$$

On the other hand, for any $M>0$, we consider the following set:

$$
\Omega_{M}=\left\{\omega \in \Omega: V_{\nu}\left(\mathscr{A}(\omega), \varepsilon_{0}\right) \leq M\right\} .
$$

Then, $\Omega_{M_{1}} \subset \Omega_{M_{2}}$ for any $M_{1} \leq M_{2}$ and $\Omega=\bigcup_{M>0} \Omega_{M}$. We can choose $M_{0}$ large enough such that $\mathbb{P}\left(\Omega_{M}\right)>0$ for all $M \geq$ $M_{0}$. It follows from the Poincaré recurrence theorem (see [13]) that, for every element $\omega \in \Omega_{M}\left(M \geq M_{0}\right)$, there exists a sequence $k_{j}=k_{j}(\omega) \rightarrow \infty$ such that $\theta_{-k_{j}} \omega \in \Omega_{M}(M \geq$ $\left.M_{0}\right)$. Therefore, from (21), for all $\omega \in \Omega_{M}\left(M \geq M_{0}\right)$,

$$
V_{\nu}\left(\mathscr{A}(\omega), q^{k_{j}} \varepsilon_{0}\right) \leq \kappa^{k_{j} \nu} V_{\nu}\left(\mathscr{A}\left(\theta_{-k_{j}} \omega\right), \varepsilon_{0}\right) \leq M \kappa^{k_{j} \nu} .
$$

From (24) we see that $q^{k_{j}} \varepsilon_{0}$ satisfies the assumptions of Lemma 2.2 in [13]. Then, (26) and the related result in [13] yield that

$$
\operatorname{dim}_{f} \mathscr{A}(\omega) \leq \nu, \quad \omega \in \Omega_{M}\left(M \geq M_{0}\right) .
$$

Since $P\left(\Omega_{M}\right) \rightarrow 1$ as $M \rightarrow \infty$, this yields that the above inequality holds for $\mathbb{P}$-a.s. $\omega \in \Omega$. The proof is complete.

As in the deterministic case [14], the following result can be easily deduced by Theorem 4 .

Theorem 5. Let $X$ and $Y$ be Banach spaces such that $Y$ is compactly embedded in $X$. Let $\mathscr{A}(\omega)$ be a compact measurable set invariant under $S(\omega)$. Assume that, for $\mathbb{P}$-a.s. $\omega \in \Omega$,

$$
\left\|S(\omega) v_{1}-S(\omega) v_{2}\right\|_{Y} \leq L\left\|v_{1}-v_{2}\right\|_{X}, \quad v_{1}, v_{2} \in \mathscr{A}(\omega),
$$

where $L$ is a constant independent of $\omega$. Then $\mathscr{A}(\omega)$ has finite fractal dimension in $X$ and admits the estimate

$$
\operatorname{dim}_{f} \mathscr{A}(\omega) \leq \ln m_{Y, X}(8 e L), \quad \mathbb{P} \text {-a.s. } \omega,
$$

where $m_{Y, X}(R)$ is the maximal number of points $x_{i}$ in the ball of the radius $R$ in $Y$ possessing the properties $\left\|x_{i}-x_{j}\right\|_{X}>1$, $i \neq j$.

Remark 6. Recalling that we have defined $n(M, \varepsilon)$ in Definition 1, we call $\mathscr{H}_{\varepsilon}(M):=\log _{2} n(M, \varepsilon)$ the Kolmogorov $\varepsilon$-entropy of $M$. Then the number $m_{Y, X}(R)$ can be bounded by the Kolmogorov entropy. To show this we assume that $i$ is the compact embedding of $Y$ into $X$ in Theorem 5 and denote 
by $B_{Y}(R)$ the ball of the radius $R$ in $Y$. By the definition of $m_{Y, X}(R)$, one can easily show that

$$
m_{Y, X}(R) \leq n\left(i B_{Y}(R), \frac{1}{2}\right) .
$$

That is,

$$
m_{Y, X}(R) \leq 2^{\mathscr{H}_{1 / 2}\left(i B_{Y}(R)\right)}=2^{\mathscr{H}_{1 / 2}\left(R i B_{Y}\right)}=2^{\mathscr{H}_{1 / 2 \mathrm{R}}\left(i B_{Y}\right)},
$$

where $B_{Y}=B_{Y}(1)$. Moreover, the Kolmogorov entropy is closely related to the entropy numbers $e_{k}(i), k \in \mathbb{N}$ (see definition in [17]). Thus, one can estimate $m_{Y, X}(R)$ by using the entropy numbers, and here we omit the details. We refer the readers to [17] for more details about the entropy numbers $e_{k}(i)$.

In the concrete application of Theorems 4 and 5, one can define

$$
S(\omega)=\phi\left(T^{*}, \omega\right) \quad \text { for some } T^{*}>0,
$$

where $T^{*}$ is independent of $\omega$ and $\phi(t, \omega)$ is an RDS on $X$ over an $\operatorname{MDS}\left(\Omega, \mathscr{F}, \mathbb{P},\left(\theta_{t}\right)_{t \in \mathbb{R}}\right)$. Then from the cocycle property we have

$$
\begin{aligned}
S^{n}(\omega) & :=\phi\left(n T^{*}, \omega\right) \\
& =\phi\left(T^{*}, \theta_{(n-1) T^{*}} \omega\right) \circ \phi\left(T^{*}, \theta_{(n-2) T^{*}} \omega\right) \circ \cdots \circ \phi\left(T^{*}, \omega\right) \\
& =S\left(\theta_{(n-1) T^{*}} \omega\right) \circ S\left(\theta_{(n-2) T^{*}} \omega\right) \circ \cdots \circ S(\omega) \\
& =S\left(\Theta_{n-1} \omega\right) \circ S\left(\Theta_{n-2} \omega\right) \circ \cdots \circ S(\omega) .
\end{aligned}
$$

This implies that $\left\{S^{n}(\omega)\right\}_{n \in \mathbb{N}}$ is a discrete RDS over the MDS $\left(\Omega, \mathscr{F}, \mathbb{P},\left(\Theta_{n}\right)_{n \in \mathbb{Z}}\right)$ on $X$, where

$$
\Theta_{n}(\omega)=\theta_{n T^{*}}(\omega) .
$$

\section{Applications}

Our abstract results can be applied to many stochastic models. In this section, we consider the following stochastic semilinear degenerate parabolic equation with variable, nonnegative coefficients defined on an arbitrary domain (bounded or unbounded) $D \subset \mathbb{R}^{N}$ with $N \geq 2$ (we refer the reader to [9] for more details):

$$
\begin{gathered}
d u+[-\operatorname{div}(\sigma(x) \nabla u)+\lambda u+f(u)] d t=\sum_{j=1}^{m} h_{j} d w_{j} \\
\text { in } D \times \mathbb{R}^{+} ; \\
u(x, t)=0 \quad \text { on } \partial D \times \mathbb{R}^{+} ; \\
u(x, 0)=u_{0}(x), \quad \text { in } D,
\end{gathered}
$$

where $\lambda>0$ and the nonlinear term $f \in C^{1}(\mathbb{R}, \mathbb{R})$ satisfies the following assumptions:

$$
f(0)=0, \quad f^{\prime}(s) \geq-l, \quad \forall s \in \mathbb{R},
$$

with positive constant $l$.
(1) The case when $D$ is bounded is as follows:

$$
\begin{gathered}
\alpha_{1}|s|^{p}-\beta_{1} \leq f(s) s \leq \alpha_{2}|s|^{p}-\beta_{2}, \quad \forall s \in \mathbb{R}, \\
\text { with positive constants } \alpha_{1}, \alpha_{2}, \beta_{1} \text {, and } \beta_{2} .
\end{gathered}
$$

(2) The case when $D$ is unbounded is as follows:

$$
\begin{gathered}
f(x, s) s \geq \alpha_{1}|s|^{p}-k_{1}(x), \\
|f(x, s)| \leq \alpha_{2}|s|^{p-1}+k_{2}(x),
\end{gathered}
$$

with positive constants $\alpha_{1}$ and $\alpha_{2}$, and $k_{1} \in L^{1}(D) \cap L^{\infty}(D)$, and $k_{2} \in L^{2}(D) \cap L^{q}(D)$, where $(1 / p)+(1 / q)=1$.

The degeneracy of problem (35) is considered in the sense that the measurable, nonnegative diffusion coefficient $\sigma(x)$ is allowed to have at most a finite number of essential zeros. We assume that the function $\sigma: D \rightarrow \mathbb{R}^{+} \cup\{0\}$ satisfies the following assumptions:

$\left(\mathscr{H}_{\alpha}\right) \sigma \in L_{\text {loc }}^{1}(D)$ and, for some $\alpha \in(0,2)$, $\lim \inf _{x \rightarrow z}|x-z|^{-\alpha} \sigma(x)>0$ for every $z \in \bar{D}$, when the domain $D$ is bounded;

$\left(\mathscr{H}_{\alpha, \beta}^{\infty}\right) \sigma \quad$ satisfies condition $\mathscr{H}_{\alpha}$ and $\lim \inf _{|x| \rightarrow \infty}|x|^{-\beta} \sigma(x)>0$ for some $\beta>2$, when the domain $D$ is unbounded.

We use the natural energy space $\mathscr{D}_{0}^{1,2}(D, \sigma)$ defined as the closure of $C_{0}^{\infty}$ with respect to the norm:

$$
\|u\|_{\mathscr{D}_{0}^{1,2}(D, \sigma)}:=\left(\int_{D} \sigma(x)|\nabla u|^{2} d x\right)^{1 / 2} .
$$

The space $\mathscr{D}_{0}^{1,2}(D, \sigma)$ is a Hilbert space with respect to the scalar product:

$$
(u, v)_{\sigma}:=\int_{D} \sigma(x) \nabla u \nabla v d x .
$$

Moreover, $\mathscr{D}_{0}^{1,2}(D, \sigma) \hookrightarrow L^{2}(D)$ compactly for both bounded (when assumption $\mathscr{H}_{\alpha}$ holds true) and unbounded (when assumption $\mathscr{H}_{\alpha, \beta}^{\infty}$ holds true) domain $D$.

We consider the following parameterized evolution equation:

$$
\frac{\partial v}{\partial t}+A v+\lambda v+f\left(v+z\left(\theta_{t} \omega\right)\right)=-A z\left(\theta_{t} \omega\right)
$$

where $v(t)=u(t)-z\left(\theta_{t} \omega\right)$ and $u(t)$ is a solution of (35). Also $A v:=-\operatorname{div}(\sigma(x) \nabla v)$ and $z$ is an Ornstein-Uhlenbeck process.

We denote by $\phi\left(t, \omega, u_{0}\right)=u\left(t, \omega, u_{0}\right)$ the RDS generated by (35) and $\mathscr{A}(\omega)$ the random attractor in $L^{2}(D)$ for the RDS $\phi$. We now verify the compact Lipschitz condition (28) as follows.

Lemma 7. Under the assumptions (36), (37), and $\mathscr{H}_{\alpha}$ for bounded domain ((36), (38), and $\mathscr{H}_{\alpha, \beta}^{\infty}$ hold for unbounded domain, resp.), one has that, for any $t \geq 1$ and $\mathbb{P}$-a.s. $\omega \in \Omega$,

$$
\begin{array}{r}
\left\|\phi\left(t, \omega, u_{0,1}\right)-\phi\left(t, \omega, u_{0,2}\right)\right\|_{\mathscr{D}_{0}^{1,2}(D, \sigma)} \leq c e^{c t}\left\|u_{0,1}-u_{0,2}\right\|, \\
\forall u_{0,1}, u_{0,2} \in L^{2}(D),
\end{array}
$$

where $c$ is independent of $\omega$. 
Proof. Setting $v_{0,1}=u_{0,1}-z(\omega)$ and $v_{0,2}=u_{0,2}-z(\omega)$, we assume that $v_{1}(t)$ and $v_{2}(t)$ are two solutions of (41) with the initial functions $v_{0,1}$ and $v_{0,2}$, respectively. We consider the difference $w(t)=v_{1}(t)-v_{2}(t)$, and then $w(t)$ satisfies

$$
w_{t}(t)+A w(t)+\lambda w(t)+l(t, \omega) w(t)=0,
$$

where $l(t, \omega)=\int_{0}^{1} f^{\prime}\left[s\left(v_{1}(t, \omega)+z\left(\theta_{t} \omega\right)\right)+(1-s)\left(v_{2}(t, \omega)+\right.\right.$ $\left.\left.z\left(\theta_{t} \omega\right)\right)\right] d s$. to get

We first take the inner product of (43) with $w(t)$ in $L^{2}(D)$

$$
\frac{1}{2} \frac{d}{d t}\|w\|^{2}+\|w\|_{\mathscr{D}_{0}^{1,2}(D, \sigma)}^{2}+\lambda\|w\|^{2}+(l(t, \omega) w, w)=0 .
$$

Also $f^{\prime}(u) \geq-l$ implies that $l(t, \omega) \geq-l$, and we can get from the above equation that

$$
\frac{d}{d t}\|w\|^{2}+2\|w\|_{\mathscr{D}_{0}^{1,2}(D, \sigma)}^{2}+2 \lambda\|w\|^{2} \leq c\|w\|^{2} .
$$

It is easy to deduce from (45) that

$$
\|w(t)\|^{2} \leq e^{c t}\|w(0)\|^{2}, \quad t \geq 0 .
$$

For any $t \geq 0$, we integrate $(45)$ into $(t, t+1)$ to get

$$
\int_{t}^{t+1}\|w(s)\|_{\mathscr{D}_{0}^{1,2}(D, \sigma)}^{2} d s \leq c\|w(t)\|^{2}+c \int_{t}^{t+1}\|w(s)\|^{2} d s .
$$

Putting (46) into the above inequality we obtain that

$$
\int_{t}^{t+1}\|w(s)\|_{\mathscr{D}_{0}^{1,2}(D, \sigma)}^{2} d s \leq c e^{c t}\|w(0)\|^{2} .
$$

Next, we multiply (43) by $A w$, and we have

$$
\begin{gathered}
\frac{1}{2} \frac{d}{d t}\|w\|_{\mathscr{D}_{0}^{1,2}(D, \sigma)}^{2}+\|A w\|^{2}+\lambda\|w\|_{\mathscr{D}_{0}^{1,2}(D, \sigma)}^{2} \\
+(l(t, \omega) w, A w)=0 .
\end{gathered}
$$

This implies that

$$
\frac{d}{d t}\|w(t)\|_{\mathscr{D}_{0}^{1,2}(D, \sigma)}^{2} \leq c\|w(t)\|_{\mathscr{D}_{0}^{1,2}(D, \sigma)}^{2} .
$$

Applying the uniform Gronwall lemma (noting that the uniform Gronwall inequality also holds true when the righthand side of (48) is dependent on $t$ !), it yields that

$$
\|w(t)\|_{\mathscr{D}_{0}^{1,2}(D, \sigma)}^{2} \leq c e^{c t}\|w(0)\|^{2} .
$$

Finally, using the relationship $u\left(t, \omega, u_{0}\right)=v\left(t, \omega, v_{0}\right)+$ $z\left(\theta_{t} \omega\right)$, one can easily deduce the result. This completes the proof.

Choosing $t=1$ in Lemma 7 and setting $S(\omega)=\phi(1, \omega)$, we see that $S(\omega)$ satisfies the assumptions in Theorem 5 with $X=L^{2}(D)$ and $Y=\mathscr{D}_{0}^{1,2}(D, \sigma)$. Therefore, we have the following.

Theorem 8. Let the assumptions of Lemma 7 hold. Then the random attractor $\mathscr{A}(\omega)$ for the RDS $\phi$ has finite fractal dimension in $L^{2}(D)$; that is, for $\mathbb{P}$-a.s. $\omega \in \Omega$,

$$
\operatorname{dim}_{f} \mathscr{A}(\omega) \leq c
$$

where $c$ is a constant independent of $\omega$.

\section{Acknowledgments}

The authors are grateful to the anonymous referees for helpful comments and suggestions that greatly improved the presentation of this paper. The paper was supported by NSF of China 10871078 and FRF for the Central Universities of China 2012 QN034 and 2013ZZGH027.

\section{References}

[1] A. V. Babin and M. I. Vishik, Attractors of Evolution Equations, vol. 25, North-Holland, Amsterdam, The Netherlands, 1992.

[2] J. K. Hale, Asymptotic Behavior of Dissipative Systems, vol. 25, American Mathematical Society, Providence, RI, USA, 1988.

[3] R. Temam, Infinite-Dimensional Dynamical Systems in Mechan$i c s$ and Physics, vol. 68, Springer, New York, NY, USA, 2nd edition, 1997.

[4] H. Crauel and F. Flandoli, "Attractors for random dynamical systems," Probability Theory and Related Fields, vol. 100, no. 3, pp. 365-393, 1994.

[5] P. W. Bates, K. Lu, and B. Wang, "Random attractors for stochastic reaction-diffusion equations on unbounded domains," Journal of Differential Equations, vol. 246, no. 2, pp. 845-869, 2009.

[6] H. Crauel, A. Debussche, and F. Flandoli, "Random attractors," Journal of Dynamics and Differential Equations, vol. 9, no. 2, pp. 307-341, 1997.

[7] T. Caraballo, J. A. Langa, and J. C. Robinson, "Stability and random attractors for a reaction-diffusion equation with multiplicative noise," Discrete and Continuous Dynamical Systems, vol. 6, no. 4, pp. 875-892, 2000.

[8] P. E. Kloeden and J. A. Langa, "Flattening, squeezing and the existence of random attractors," Proceedings of the Royal Society of London A, vol. 463, no. 2077, pp. 163-181, 2007.

[9] M. Yang and P. E. Kloeden, "Random attractors for stochastic semi-linear degenerate parabolic equations," Nonlinear Analysis: Real World Applications, vol. 12, no. 5, pp. 2811-2821, 2011.

[10] H. Crauel and F. Flandoli, "Hausdorff dimension of invariant sets for random dynamical systems," Journal of Dynamics and Differential Equations, vol. 10, no. 3, pp. 449-474, 1998.

[11] A. Debussche, "On the finite dimensionality of random attractors," Stochastic Analysis and Applications, vol. 15, no. 4, pp. 473491, 1997.

[12] A. Debussche, "Hausdorff dimension of a random invariant set," Journal de Mathématiques Pures et Appliquées, vol. 77, no. 10, pp. 967-988, 1998.

[13] J. A. Langa and J. C. Robinson, "Fractal dimension of a random invariant set," Journal de Mathématiques Pures et Appliquées, vol. 85, no. 2, pp. 269-294, 2006.

[14] I. Chueshov and I. Lasiecka, "Long-time behavior of second order evolution equations with nonlinear damping," in Memoirs of the American Mathematical Society, vol. 195, American Mathematical Society, Providence, RI, USA, 2008.

[15] K. Falconer, Fractal Geometry, John Wiley \& Sons, Chichester, UK, 1990.

[16] L. Arnold, Random Dynamical Systems, Springer, Berlin, Germany, 1998.

[17] D. E. Edmunds and H. Triebel, Function Spaces, Entropy Numbers, Differential Operators, vol. 120 of Cambridge Tracts in Mathematics, Cambridge University Press, New York, NY, USA, 1996. 


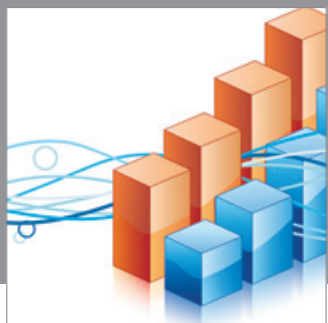

Advances in

Operations Research

mansans

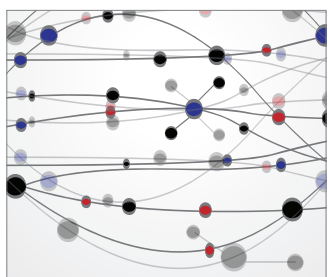

The Scientific World Journal
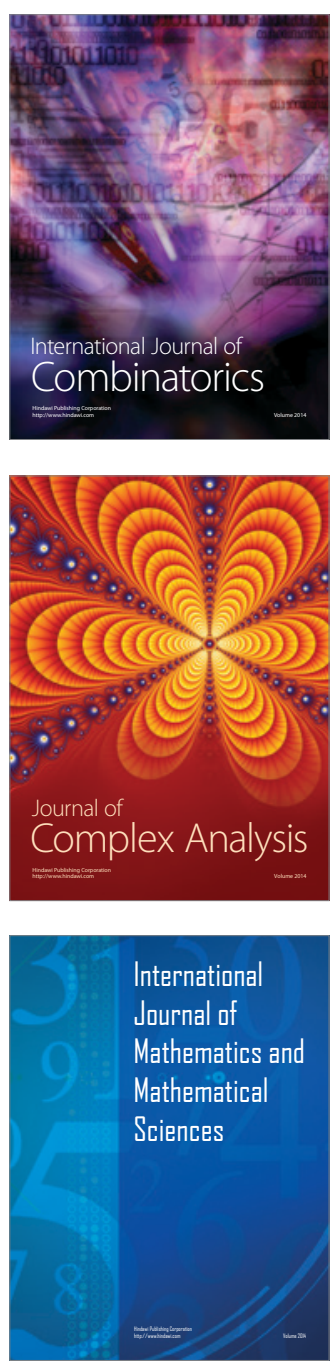
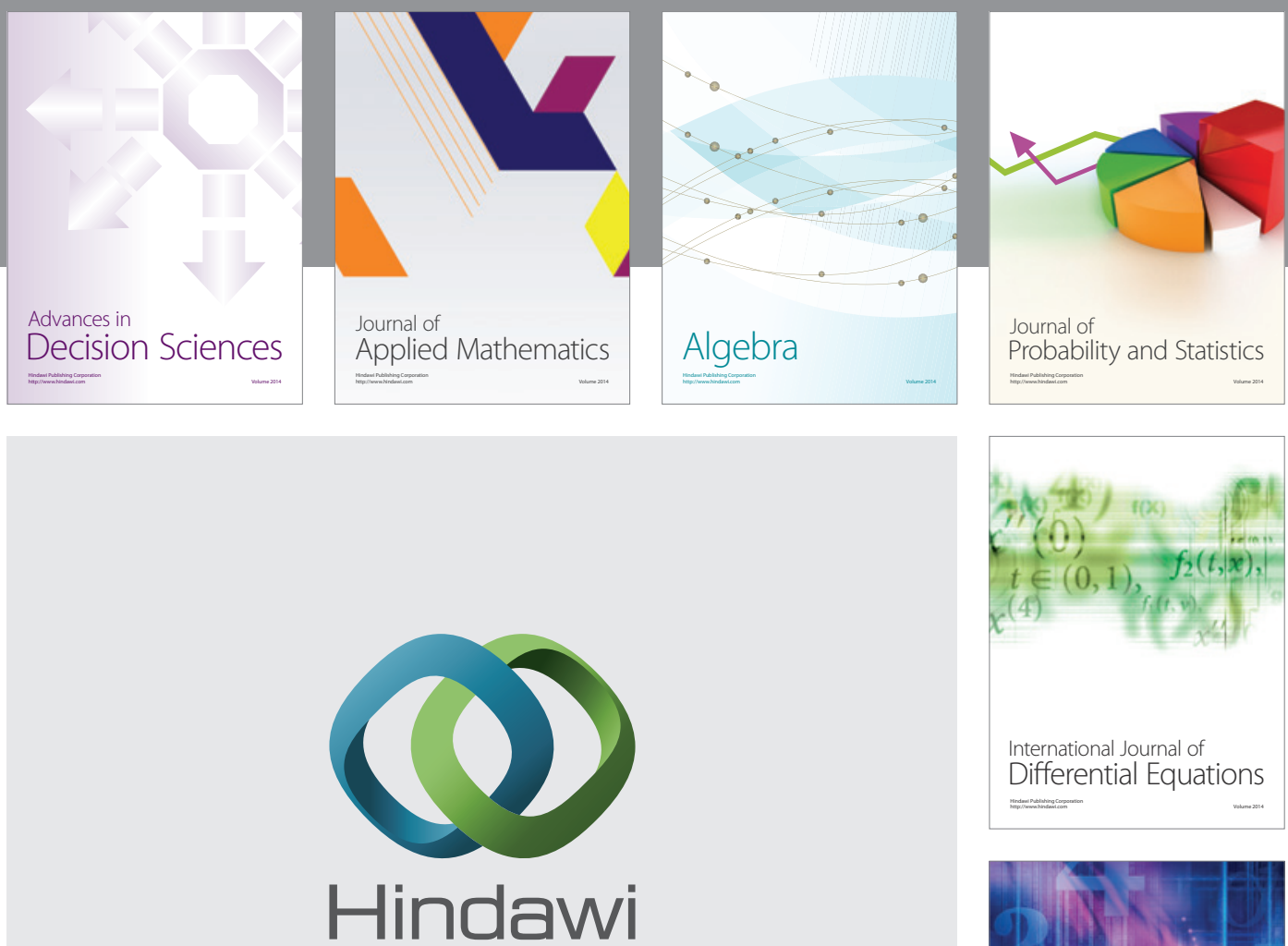

Submit your manuscripts at http://www.hindawi.com
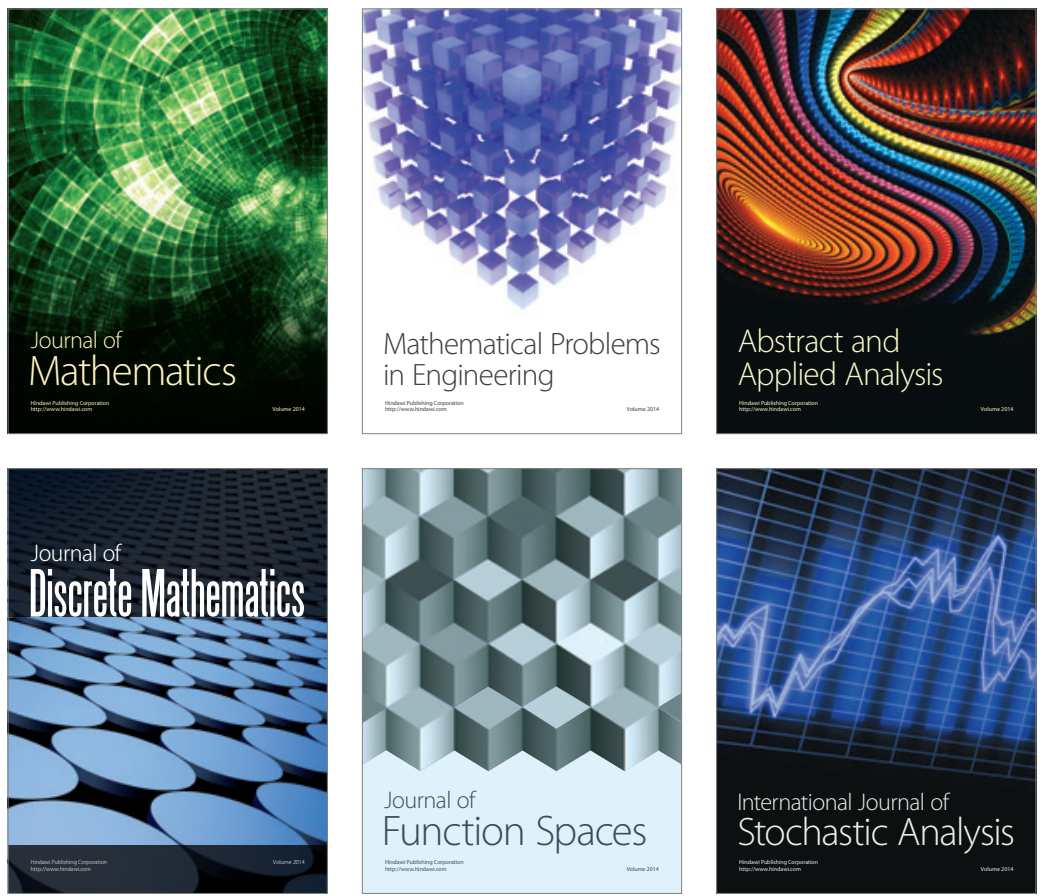

Journal of

Function Spaces

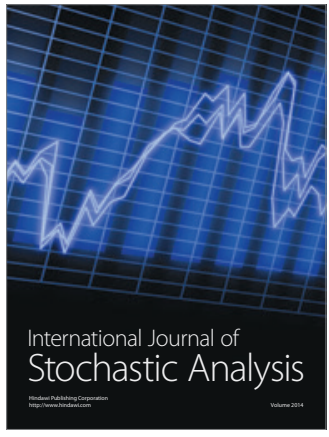

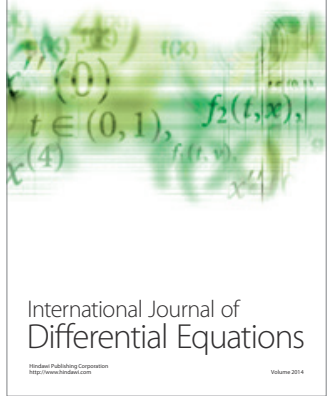
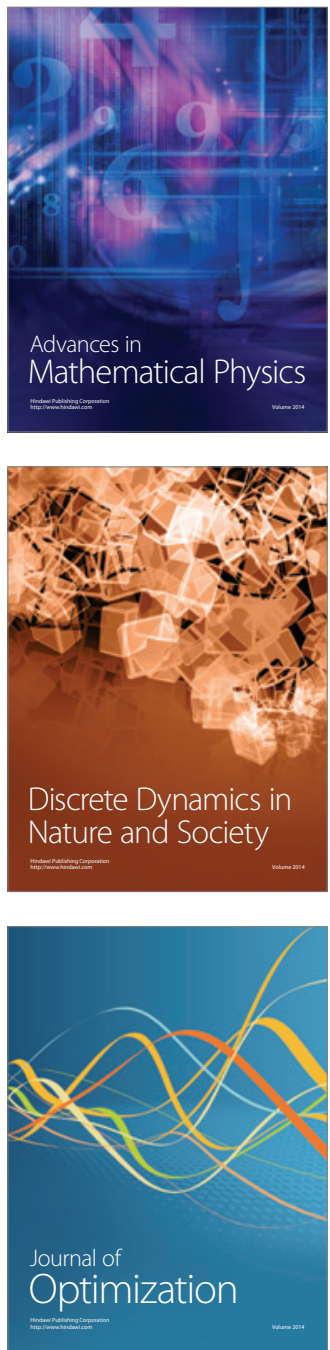